\title{
Surgery and functional mobility: doing the right thing at the right time
}

\author{
J. Dronkers ${ }^{1,2} \cdot$ B. Witteman ${ }^{3,4} \cdot$ N. van Meeteren ${ }^{5,6}$
}

Received: 29 April 2016/Accepted: 29 April 2016/Published online: 11 May 2016

(C) Springer-Verlag Italia Srl 2016

The pace of change in Western societies is rapid, and this includes change in the healthcare sector which is initiated directly and indirectly by technological and sociocultural innovation. Here, the classic dominant medical approach is complemented by an orientation toward functioning (in line with the WHO International Classification of Functioning, Disability and Health 2002). This more dual orientation is also useful during major life events, like hospital admission for surgery. Anesthetic and surgical state-of-the-art techniques will normally be adequate for tackling the medical problems for which patients were admitted. However, one of the major common side effects, functional decline, before (in the "waiting" period), during and after hospitalization is impressive, especially in old and frail people, and needs complementary prevention and care interventions [1]. Firstly, older adults have a lower muscle mass at admission or are even in a sarcopenic state. Subsequently, surgery itself, especially major abdominal, thoracic and orthopedic surgery, severely challenges the

J. Dronkers

jaap.dronkers@hu.nl

1 Department of Physical Therapy, Gelderse Vallei Hospital, Willy Brandtlaan 10, Ede, The Netherlands

2 Faculty of Healthcare, University of Applied Sciences Utrecht, Utrecht, The Netherlands

3 Department of Gastroenterology and Hepatology, Gelderse Vallei Hospital, Ede, The Netherlands

4 Division of Human Nutrition, Wageningen University, Wageningen, The Netherlands

5 Health $\sim$ Holland, Topsector Life Sciences and Health, The Hague, The Netherlands

6 CAPHRI, Maastricht University, Maastricht, The Netherlands psychophysiological system. The surgical stress response encompasses a wide range of physiological effects, which seriously and directly impair cardiopulmonary and muscle function [2]. Hormonal dysregulation and the inflammatory response contribute to an accelerated loss of lean body tissue. On top of this, post-surgery "activities" like bed rest, still the prevailing and dominant hospital recovery strategy, contribute to a progressive loss of functional capacity via a loss of (lower) extremity strength, power and aerobic capacity. In elderly patients, bed rest induces an approximate three- to sixfold greater rate of muscle mass loss compared to younger, fit persons. In addition, surgery causes three times more muscle mass loss than hospitalizations without surgery [3]. These notions call for action, and this editorial will discuss what types of action.

Impaired functioning is a frequently published serious side effect of surgery, but has increasingly been brought to notice in recent decades as life expectancy increased rapidly and more and more elderly had indications for surgery. Covinsky [4] labeled surgical functional decline as "hospitalization associated disability," In accordance with Covinsky, Lawrence Lee stated: "focusing only on the physical domain and ignoring the other domains will incorrectly describe this patient as 'recovered' from surgery" [5]. Recently, hospitalization-associated disability has been recognized as an iatrogenic but preventable disorder [6]. Prevention of a complicated (post)operative course and a swift return to an adequate performance of activities of daily living (ADL) and instrumental ADL (IADL) in older patients is mandatory and essential to preserve independent functioning and quality of life. This will limit direct care costs and additional costs for home care or even admission to a nursing home.

The first step in preventive care includes preoperative screening of the patient in order to determine the potential 
risks for functional decline. Preoperative cardiorespiratory fitness, muscle strength and performance of physical activities proved to be independent risk factors for postoperative complications, recovery of functioning and morbidities and mortality in major abdominal and thoracic surgery [7]. These factors are indicators of the adaptive capacity of the patient (homeostasis and adequacy of responses to allostatic load) to cope with the psychophysiological consequences of surgery. The specific composition of indicators differs per type of surgical procedure. Outpatient preoperative screening thus aims to identify as soon as possible those individual patients that are at risk of a complicated postoperative course. Screening should integrate preoperative physical fitness and physical activity level indicators, besides the well-known personal and/or medical factors such as age, nutrition, mental health and comorbidities (like diabetes, chronic obstructive pulmonary disease or heart failure).

Preoperative risk identification by screening enables patients and physiotherapists to jointly reflect on the postoperative risk in order to optimize patients' preoperative physical condition and adaptive capacity before planning and execution of elective surgery. In case of high risks, preoperative prophylactic exercise training is offered to the patient. This appears to be beneficial to older, especially frail high-risk individuals. Their improved preoperative adaptive capacity helps them to better withstand the consequences of surgery and hospitalization in the postoperative period. However, in daily practice, the period available for prophylactic exercise training before elective surgery may seem limited, especially with oncological abdominal and thoracic surgery where medical urgency should be weighed against the fitness of each patient. In recent years, a number of reviews have been published about the effect of short-term preoperative training programs [8]. The overall conclusion is that preoperative training is feasible in the young, the old and the very old, and even the old and frail. The reviews provide evidence for the improvement of preoperative physical fitness measures. However, they also show a substantial variance in type and intensity of the training programs.

The type of training depends on a clear rationale which provides patients and their physical therapists with the means for making valid choices and shared decisions regarding the type of training and the goals to strive for. For example, an adequate physical fitness program is proposed to guarantee early postoperative ambulation, which in itself already prevents sedentary behavior. Consequently, part of the hospitalization-induced functional decline and postoperative complications are prevented. According to such a rationale, preoperative prophylactic exercise training should be directed to the required fitness for ADL and the ADL itself. Because functional mobility
ADL is closely related to lower leg muscle function, the functional activities in the training program should focus on these muscles. Preoperative training of patients' cardiorespiratory fitness seems to prepare them for the increased metabolic demands after surgery. Likewise, the rationale of the adaptive capacity of the immune response is of interest here. This rationale could explain the importance of the degree of physical activity which shows an U-shaped dose response curve with immunity [9].

Due to the limited time for training before surgery, the intensity of the training has to be high enough to achieve short-term improvements. Frail older patients can cope with and tolerate high-intensity training, but they also require a longer recovery period than younger people. Thus, overtraining lurks in ambush and should be avoided, because this puts the patient in an even worse preoperative condition than an absence of training would. Research and especially "titration" of dose-response monitoring should help to optimize the training parameters, aiming at maximal improvement without overtraining, which is a real hazard for frail older people [10].

A patient's compliance with a training program may be inversely related to the intensity of the program, so that to keep the patient on board, the intensity level should not always be maximal. Home-based physiotherapist-supervised training has been shown to enhance the compliance of very frail patients, improving the effectiveness of the training program. What is more, the patient's relatives involve, activate, motivate and support their loved ones, even when the therapist is not around. On top of this, jointly monitoring a patient's responses and progression during the training program and discussing these results with the patient, therapist and, if allowed, the patient's (in)formal caregivers will improve the patient's motivation and compliance [10].

Recently, this journal published a systematic review by Boereboom et al. who also concluded that further investigation of different forms of exercises is required. In this issue of the journal, Boereboom reports evidence for physiotherapist-supervised high-intensity interval training in the outpatient clinics.

The study is a precursor to a more extensive study with a colorectal cancer patient population. This is a relevant target population because colorectal cancer patients may have a higher risk profile as a result of a higher incidence of weight loss due to malnutrition or diarrhea. Moreover, postoperative ileus or bowel dysfunction may further worsen the postoperative course [11].

The outcome measures of Boereboom's study cover both the cardiorespiratory and muscle components. High intensity is combined with interval training, which can promote compliance. However, we have to take into consideration that this research included fairly healthy 
individuals. For colorectal cancer patients, compliance can be enhanced by offering the training in a home-based setting. We also would like to motivate Boereboom and colleagues to incorporate the preoperative screening and to apply the training in trials to high-risk patients.

Boereboom makes use of a quasi-experimental design, without a control group, which hampers external validity to a certain extent. But, it should be debated whether or not randomized controlled trials (RCT) are appropriate, considering that nowadays knowledge and rationales about perioperative care are already available. From a methodological point of view, a well-powered RCT should provide the missing evidence and include postoperative outcomes. But the patients (involve them!), researchers and clinicians should question whether the performance of a RCT (including withholding of intervention) is still an ethically sound decision. The strong evidence for the relationship between preoperative physical activity and physical fitness and the patient's postoperative course, as well as the biological plausibility (showing a relationship between the physical condition and the adaptive capacity of the body system), raises the ethical question whether new RCT research with the use of a control group without any form of intervention is justified or not.

This issue addresses the therapeutic obligations of patients and clinicians, and makes the, probably artificial and unwanted, distinction between a research and real-life clinical context approach apparent. The literature proposes "clinical equipoise," as a requirement for random assignment of patients. It is defined as a state of professional uncertainty about their relative therapeutic merits and is a much-debated issue. We should find out whether there is sufficient disagreement or absence of agreement among patients and expert clinicians about the relative merits of the alternatives of preoperative training. Besides, it is possible to make use of research designs other than RCT, such as a stepped wedge design, comparative effectiveness research (CER) and others, for instance, naturalistic trials [12].

Together with other research in this field, the study of Boereboom et al. contributes to an approach that should, in the near future, optimize successful outcomes of surgery, with physical fitness brought into preoperative care as a patient's predictive, modifiable and trainable variable.

\section{Compliance with ethical standards}

Conflict of interest The authors declare that they have no conflict of interest.

Ethical approval The present paper was written according to an appropriate ethical standards.

Informed consent For this editorial informed consent is not required.

\section{References}

1. Hulzebos EH, van Meeteren NL (2016) Making the elderly fit for surgery. Br J Surg 103:e12-e15

2. Desborough JP (2000) The stress response to trauma and surgery. Br J Anaesth 85:109-117

3. English KL, Paddon-Jones D (2010) Protecting muscle mass and function in older adults during bed rest. Curr Opin Clin Nutr Metab Care 13:34-39

4. Covinsky KE, Pierluissi E, Johnston CB (2011) Hospitalizationassociated disability: "She was probably able to ambulate, but I'm not sure". JAMA 306:1782-1793

5. Lee L, Tran T, Mayo NE, Carli F, Feldman LS (2014) What does it really mean to "recover" from an operation? Surgery 155:211-216

6. Sourdet S, Lafont C, Rolland Y, Nourhashemi F, Andrieu S, Vellas B (2015) Preventable iatrogenic disability in elderly patients during hospitalization. J Am Med Dir Assoc 16:674-681

7. Moran J, Wilson F, Guinan E, McCormick P, Hussey J, Moriarty J (2016) Role of cardiopulmonary exercise testing as a riskassessment method in patients undergoing intra-abdominal surgery: a systematic review. Br J Anaesth 116:177-191

8. Santa Mina D, Clarke H, Ritvo P et al (2014) Effect of total-body prehabilitation on postoperative outcomes: a systematic review and meta-analysis. Physiotherapy 100:196-207

9. Romeo J, Warnberg J, Pozo T, Marcos A (2010) Physical activity, immunity and infection. Proc Nutr Soc 69:390-399

10. Glasziou P, Irwig L, Mant D (2005) Monitoring in chronic disease: a rational approach. BMJ 330:644-648

11. Martindale RG, McClave SA, Taylor B, Lawson CM (2013) Perioperative nutrition: what is the current landscape? JPEN J Parenter Enteral Nutr 37(5 Suppl):5S-20S

12. Craig P, Cooper C, Gunnell D et al (2012) Using natural experiments to evaluate population health interventions: new Medical Research Council guidance. J Epidemiol Community Health 66:1182-1186 